\title{
Patient considerations in cataract surgery - the role of combined therapy using phenylephrine and ketorolac
}

This article was published in the following Dove Press journal:

Patient Preference and Adherence

15 September 2016

Number of times this article has been viewed

\author{
Roberto Gonzalez-Salinas ${ }^{1,2}$ \\ Adriano Guarnieri ${ }^{3}$ \\ María Concepción Guirao \\ Navarro ${ }^{3}$ \\ Manuel Saenz-de-Viteri ${ }^{3}$ \\ 'Department of Biomedical Research, \\ Universidad Autónoma de Querétaro, \\ Querétaro, Mexico; ${ }^{2}$ Department \\ of Research, Asociación para Evitar \\ la Ceguera en México, Mexico \\ City, Mexico; ${ }^{3}$ Department of \\ Ophthalmology, Clínica Universidad \\ de Navarra, Pamplona, Spain
}

\begin{abstract}
Cataract, a degradation of the optical quality of the crystalline lens, progressive and age-related, is the leading cause of treatable blindness worldwide. Cataract surgery is the most common surgical procedure performed by ophthalmologists and is the only effective treatment for cataracts. Advances in the surgical techniques and better postoperative visual outcomes have progressively changed the primary concern of cataract surgery to become a procedure refined to yield the best possible refractive results. Sufficient mydriasis during cataract removal is critical to a successful surgical outcome. Poor pupil dilation can lead to serious sight-threatening complications that significantly increase the cost of surgery and decrease patients comfort. Mydriasis is obtained using anticholinergic and sympathomimetic drugs. Phenylephrine, an $\alpha 1$-adrenergic receptor agonist, can efficiently dilate the pupil when administered by intracameral injection. Additionally, nonsteroidal anti-inflammatory drugs (NSAIDs) like ketorolac, which inhibit the synthesis of prostaglandins, are used to decrease intraoperative miosis, control pain and inflammation associated with cataract surgery, and to prevent the development of cystoid macular edema following surgery. Recently, a new combination of phenylephrine and ketorolac $\left(\right.$ Omidria $\left.^{\circledR}\right)$ has been approved by United States Food and Drug Administration for use during cataract surgery to maintain intraoperative mydriasis, prevent miosis, and reduce postoperative pain and inflammation. Clinical trials have shown that this new combination is effective, combining the positive effects of both drugs with a good safety profile and patient tolerability. Moreover, recent reports suggest that this combination is also effective in patients with high risk of poor pupil dilation. In conclusion, cataract is a global problem that significantly affects patients' quality of life. However, they can be managed with a safe and minimally invasive surgery. Advances in surgical techniques and newer pharmacological agents such as the combination of phenylephrine and ketorolac, together with better intraocular lenses, have greatly improved visual outcomes and thus patients' expectations regarding visual recovery are also increasing.
\end{abstract}

Keywords: cataract surgery, mydriasis, phenylephrine, ketorolac, Omidria ${ }^{\circledR}$

\section{Introduction - management of cataract surgery and patient considerations}

A cataract is a degradation of the optical quality of the crystalline lens. It is usually a progressive, chronic, age-related disease affecting a large number of people above the age of 50 and is characterized by clouding of the lens of the eye, which prevents the light from reaching the retina, thus impeding clear vision. ${ }^{1,2}$ Although most cases are related to the aging process, occasionally children can be born with this condition, or a cataract may develop after eye injuries, inflammation, and some other ocular
Correspondence: Manuel Saenz-de-Viteri Ave Pio XII 36, 31008 Pamplona,

Navarra, Spain

Tel +34948948255400

Fax +34948296500

Email msaendevit@alumni.unav.es 
diseases. ${ }^{1,3}$ Cataracts are the leading cause of treatable blindness worldwide, accounting for more than $40 \%$ of cases. ${ }^{4}$ In addition, $75 \%$ of people with cataracts live in developing countries where blindness is associated with considerable disability and major economic and social consequences. ${ }^{5}$ Cataract surgery is the most common procedure performed by ophthalmic surgeons ${ }^{6}$ and is the only effective treatment for cataracts. According to the UK National Health Service, $\sim 330,000$ cataract surgeries are performed every year in England. ${ }^{6}$ Similarly, in the US, at least 1.8 million cataract surgeries are performed on Medicare patients every year. ${ }^{7}$ Therefore, it is important to evaluate the safety and effectiveness and ultimately the cost/effectiveness of cataract removal procedures. ${ }^{4}$

Surgical outcome criteria can vary depending on the patient's needs, lifestyle, and associated medical conditions. In general, these criteria include the reduction of visual symptoms, improvement of visual function, achievement of the desired refractive outcome, and improvement of physical function, mental health, and quality of life..$^{5,6,8}$ However, recent advances in surgical techniques in addition to the improvement of postoperative visual outcomes have progressively changed the primary concern of cataract surgery from the safe removal of the opaque crystalline lens to a procedure refined to yield the best possible refractive result. ${ }^{9}$ Emmetropia can be achieved for patients with myopic or hyperopic refractive errors and astigmatism by selecting the appropriate intraocular lens (IOL). ${ }^{10,11}$ Thus, refractive lens exchange (RLE) has become a growing modality in refractive surgery, ${ }^{12}$ especially with recent IOL technology aiming to provide distance, intermediate, and near-spectacle independence. ${ }^{13-15}$ Therefore, an uneventful cataract surgery requires an optimal level of intraoperative mydriasis throughout the procedure, ${ }^{16}$ in order to achieve a good visualization of the surgical field and to prevent possible complications with undesired postoperative symptoms. ${ }^{9}$

\section{Pre-, peri-, and postoperative considerations, optimization of procedure, and minimization of postoperative complications}

Modern cataract surgery requires a stable and adequate mydriasis lasting throughout the procedure in order to effectively perform all the steps of the surgery. The surgeon should assess the quality of dilation during the presurgical examination, and a careful medical history is important to reduce the likelihood of unexpected intraoperative miosis. ${ }^{17}$ Proper mydriasis during cataract removal and IOL placement is critical to achieve a successful surgical outcome by providing a complete visualization of the surgical field, a good red reflex, and adequate room for intraocular manipulation of surgical instruments. ${ }^{17}$ Poor pupil dilation can lead to serious sight-threatening complications such as anterior or posterior capsule tears, vitreous loss and iris trauma during surgery, and the development of cystoid macular edema (CME) or retinal detachment during the postoperative period. ${ }^{18}$

Disruption of the capsule barrier between the anterior chamber and the vitreous is a major complication of cataract surgery that might compromise the visual prognosis. ${ }^{19}$ This complication is estimated to occur in $1.9 \%-5.2 \%$ of phacoemulsification procedures. ${ }^{19,20}$ Capsule complications result in a substantial increase in additional care including more visits after the surgery, higher need for in-patient care, and a greater percentage of a second surgery. ${ }^{19}$ It is estimated that this complication can double the cost of an uneventful cataract removal procedure. ${ }^{19}$ Similarly, CME following cataract surgery, which remains the most frequent postoperative complication resulting in impaired vision, ${ }^{7,17}$ can increase the cost of cataract surgery by $40 \%-50 \%{ }^{7}$ The incidence of CME after cataract surgery varies between $0.2 \%$ and $20 \%$ depending on whether the diagnosis was confirmed by clinical examination alone or with optical coherence tomography or fluorescein angiography. ${ }^{7,21}$

Since not all cataract procedures have the same degree of complexity, a complete presurgical evaluation might benefit from scoring systems, like the one developed by Muhtaseb et $\mathrm{al}^{22}$ that allows the surgeon to categorize patients according to their risk of intraoperative complications, or the regression model proposed by Moreno-Montañés et $\mathrm{al}^{23}$ aimed to identify high-risk patients, thus allowing the cataract surgeon to anticipate potential difficulties in challenging cases.

\section{Pupil dilation and phenylephrine}

It is important to know the structure and physiology of the iris to understand how it accommodates changes in pupil diameter. The posterior iris leaf contains the dilator and the sphincter muscles, separated by a dense band of connective tissue called iris stroma. ${ }^{24}$ The dilator muscle is innervated by the sympathetic nervous system. ${ }^{25}$ Therefore, stimulation of the sympathetic fibers leads to a pupil dilation. ${ }^{26}$ The sphincter muscle, located just inside the pupillary border, is made up of $\sim 20$ motor segments connected together but innervated individually by postganglionic branches of the ciliary nerve. ${ }^{25}$ Contraction of the sphincter causes the pupil to constrict causing miosis. This muscle is innervated by the parasympathetic system. ${ }^{25}$ Therefore, blocking of the 
parasympathetic pathway with muscarinic antagonists leads to mydriasis. ${ }^{26}$ As the iris sphincter is stronger than the dilator muscle, its action will determine the pupil size under a bright microscope light. ${ }^{27}$

Mydriasis for cataract surgery is obtained using medications that elicit anticholinergic and sympathomimetic effects. Phenylephrine and tropicamide are most commonly used, either alone or in combination. ${ }^{16,28}$ Phenylephrine, an $\alpha 1$-adrenergic receptor agonist, acts as a mydriatic agent when administered to the eye by contracting the dilator muscle of the iris with 5-7 hours of dilation. ${ }^{29}$ It can be used at different concentrations $(2.5 \%, 5 \%$, and $10 \%)$ and it effectively dilates the pupil when administered by intracameral injection. ${ }^{26,27,30,31}$ Moreover, lower concentrations have been shown to be effective in maintaining mydriasis during cataract surgery, with a lower risk of systemic side effects. ${ }^{16}$ Intracameral mydriatic agents were introduced in 2003 and have been shown to provide sufficient pupil dilation without causing measurable ocular side effects or negatively influencing the surgical procedure. ${ }^{16}$

\section{Nonsteroidal anti-inflammatory drugs in cataract surgery}

NSAIDs prevent the synthesis of prostaglandins by inhibiting cyclooxygenase enzymes 1 and 2. Five ophthalmic NSAIDs are currently available for use in the US: flurbiprofen, ketorolac, diclofenac, bromfenac, and nepafenac. ${ }^{32}$ These drugs have been approved for the management of pain and inflammation associated with cataract surgery and corneal refractive surgery, inhibition of intraoperative miosis, and seasonal allergic conjunctivitis. However, their use has expanded beyond these indications, particularly for the prevention of CME following cataract surgery. . $32^{2}$

Although these five drugs share the same mechanism of action, none of the medications are approved by the US Food and Drug Administration (US FDA) for all the aforementioned indications, but their efficacy within and outside their US FDAapproved uses has been demonstrated in several studies. ${ }^{32}$

\section{Surgically induced miosis}

Prostaglandins are synthesized in the iris and ciliary body in response to trauma during anterior segment surgery. ${ }^{16,33}$ Stimulation of prostaglandin synthesis can lead to pupillary constriction, a phenomenon called surgically induced miosis. ${ }^{16,34,35}$ This decrease in pupil diameter can make cataract removal more difficult and increase the risk of surgical trauma, postoperative ocular inflammation, and posterior capsule rupture. ${ }^{18,35}$
Although flurbiprofen sodium $0.03 \%$ is the only ophthalmic NSAID approved by the US FDA for the inhibition of intraoperative miosis, several studies have compared flurbiprofen with other NSAIDs, including ketorolac, in regard to this indication. ${ }^{32,33,35,36}$

Ophthalmic ketorolac tromethamine was originally in introduced in 1992 for temporary relief of ocular itching caused by seasonal allergic conjunctivitis and for the treatment of postoperative inflammation in patients who have undergone cataract extraction. ${ }^{32,37}$ It has been demonstrated that both ketorolac $0.5 \%$ and ketorolac $0.4 \%$ are able to inhibit miosis when used preoperatively. ${ }^{32}$ When compared with flurbiprofen $0.03 \%$, studies have not found significant differences in intraoperative pupil diameter between the two drugs. ${ }^{32}$ On the contrary, when compared with diclofenac $0.1 \%$, ketorolac $0.5 \%$ was found to be more effective in maintaining mydriasis when three preoperative doses were used. ${ }^{33}$ Moreover, it has been previously theorized that intraocular mydriatics such as epinephrine in combination with topical prostaglandin inhibitors such as ketorolac may have an additional, if not a synergistic, effect on miosis inhibition during routine cataract surgery. ${ }^{33}$

\section{Pain and inflammation}

With the use of modern techniques, cataract surgery no longer requires complete ocular akinesia, thus promoting the use of less-invasive anesthetic modalities. ${ }^{38}$ Topical and intracameral anesthesia are associated with lower rates of serious complications such as retrobulbar hemorrhage, globe perforation, and traumatic injury to the optic nerve when compared to retrobulbar and peribulbar methods. ${ }^{17,39}$ However, patients under topical anesthesia may experience greater anxiety and ocular discomfort during the surgical procedure. Consequently, perioperative pain management becomes important to improve the patient's intraoperative cooperation. ${ }^{40}$ Moreover, pain during the first hours after cataract surgery is relatively common. A prospective study conducted in Finland reported that up to $34 \%$ of patients experienced ocular pain during the early postoperative period. ${ }^{41}$ For this reason, diclofenac $0.1 \%$, ketorolac $0.5 \%$, ketorolac $0.4 \%$, bromfenac $0.07 \%$, bromfenac $0.09 \%$, nepafenac $0.1 \%$, and nepafenac $0.3 \%$ are all approved by FDA for the treatment of postoperative pain and inflammation associated with cataract surgery. ${ }^{32}$

It has been emphasized again that ketorolac in different concentrations has demonstrated to efficiently reduce pain and most symptoms of inflammation in patients with moderate to severe inflammatory responses after surgery, showing 
better results when started preoperatively. ${ }^{32}$ Some studies have compared ketorolac to other NSAIDs, although there are no significant differences in the efficacy of ketorolac $0.5 \%$ and diclofenac $0.1 \%$. A study that compared ketorolac $0.4 \%$ qid with nepafenac $0.1 \%$ tid found that ketorolac achieved better pain control along with higher rates of patient satisfaction and compliance. ${ }^{32,42}$

Conventional topical NSAIDs are increasingly employed in ophthalmology for the prevention and treatment of CME associated with cataract surgery. When compared to placebo or topical corticoid formulations with poor corneal penetration, NSAIDs have demonstrated to be more effective in reducing the incidence of $\mathrm{CME}$ and hasten visual recovery in the short term ( $<3$ months). Several studies have shown that ketorolac is effective in the treatment and prevention of $\mathrm{CME}$, when used alone or in combination with corticosteroids. ${ }^{43,44}$ In a randomized, prospective study, ketorolac and diclofenac were found to be equally effective in reducing the severity and duration of CME after uncomplicated cataract surgery. ${ }^{45}$ Although rare, decreased vision associated with postsurgical CME might affect patients several months or even years after cataract surgery. Weisz et $\mathrm{al}^{46}$ recently reported that ketorolac is effective in the treatment of these uncommon cases of chronically developed CME. ${ }^{46}$

\section{Pharmacology and clinical efficacy of the phenylephrine/ketorolac combination}

Omidria $^{\circledR}$ (Omeros Corporation, Seattle, WA, USA), is a bisulfite-free and preservative-free combination of ketorolac $0.3 \%$ and phenylephrine $1 \%$ targeted for use during cataract surgery and RLE. ${ }^{47}$ This product was approved by the FDA in May 2014 and is the only FDA-approved product for intraocular use during cataract surgery to maintain intraoperative mydriasis and reduce postoperative pain and inflammation resulting from surgery. ${ }^{26,47,48}$ This combination is added to the standard balanced saline irrigation solution bottle for intracameral irrigation in the anterior chamber during ocular surgery. ${ }^{26,47,48}$ Thus, pupil dilation can be safely achieved without further pharmacological measures or the use of pupil expansion devices that may add complexity to the procedure and increase surgical time. ${ }^{47}$

A multicenter, randomized, parallel-group, doublemasked, placebo-control Phase III study was conducted in the US and the Netherlands to evaluate the effectiveness of the phenylephrine/ketorolac combination on intraoperative pupil diameter and early postoperative ocular pain. The primary endpoints of the study were observed to be changes in pupil diameter from surgical baseline to the end of surgical procedure and postoperative ocular pain (using a visual analog scale). ${ }^{47} \mathrm{~A}$ total of 406 patients were included in the study and the phenylephrine/ketorolac combination was significantly found to be superior to placebo in maintaining intraoperative mydriasis and reducing postoperative pain. In the treatment group, $95.9 \%$ of the patients had a pupil diameter $>6 \mathrm{~mm}$ at the completion of cortical clean-up, whereas this percentage was only $77.0 \%$ in the placebo group. In addition, treatment was associated with $>50 \%$ reduction of acute postoperative pain. ${ }^{47}$

These results have been confirmed by two pivotal Phase III randomized trials that included 808 patients. ${ }^{48}$ In these studies, the proportion of patients with a pupil diameter of $<6.0 \mathrm{~mm}$ at any time during surgery and intraoperative pupillary constriction $\geq 2.5 \mathrm{~mm}$ were significantly lower among treated patients $(P<0.0001$ for each endpoint $) .{ }^{48}$ Moreover, the mean area under the curve of ocular pain visual scores within 12 hours of surgery was significantly lower in patients treated with phenylephrine/ketorolac combination (4.16 $\mathrm{mm}$ vs $9.06 \mathrm{~mm}$ in the placebo group, $P<0.001) .{ }^{48}$

Similarly, Rosenberg et $\mathrm{al}^{49}$ recently reported that the phenylephrine and ketorolac combination was associated with a statistically significant reduction in surgical time, intraoperative complications, and use of pupil dilating devices as well as a significant improvement of best corrected visual acuity on postoperative day 1 . Another recent study included 46 patients with poorly dilated pupils and floppy iris syndrome and the results showed that intracameral phenylephrine/ketorolac maintained intraoperative pupil dilation without the need to insert an iris fixation ring in any patient. ${ }^{50}$ Although the sample size is small, the difference between the phenylephrine/ketorolac and intracameral epinephrine groups was statistically significant. ${ }^{50}$ Regarding ocular discomfort, Fry ${ }^{51}$ reported recently that although the use of the phenylephrine/ketorolac combination resulted in larger pupil diameters throughout the surgery, there were no differences in the comfort level, either at surgery or during the first 24 hours between treated and untreated patients. However, discomfort levels in both groups were very low and probably not high enough to show an effect from treatment. ${ }^{51}$

\section{Safety and tolerability}

The effects of topical phenylephrine have been thoroughly studied, and it has been demonstrated that although higher concentrations of this drug increase the effectiveness of pupil dilation, they might be associated with cardiovascular side effects in high-risk populations. ${ }^{16,26}$ However, the systemic 
absorption rate of intracamerally administered substances is believed to be limited by the aqueous humor turnover rate and, therefore, the risk of systemic side effects may generally be lower when compared to topical administration. ${ }^{52}$ Additionally, intracameral injection of substances with potential cardiovascular side effects has been shown to cause little or no cardiovascular side effects. ${ }^{52,53}$

Experiments in vitro have demonstrated that exposure to phenylephrine does not change the morphology and mean cellular density of corneal endothelial cells. ${ }^{54}$ A study performed in rabbits found that the injection of intracameral phenylephrine did not produce a loss of corneal endothelial cells and did not affect intraocular pressure or central corneal thickness. ${ }^{55}$ Furthermore, intracameral administration of phenylephrine does not seem to modify the reduction rate of corneal endothelial cell density at short or long term after cataract surgery. ${ }^{52}$

With regards to topical NSAIDs, they have been demonstrated to effectively maintain intraoperative mydriasis and possess a more favorable risk-benefit profile than other drugs like epinephrine or corticosteroids. ${ }^{7,35,37}$ A study performed on male New Zealand rabbits examined the concentrations of ketorolac in the ipsilateral vitreous and retina, as well as plasma concentrations after intracameral injection of the drug. Investigators found that the concentration of ketorolac in the plasma was highest 0.5 hours after intracameral injection, but the drug in the plasma was undetectable even after 4 hours. ${ }^{37}$ The authors also found that plasma concentrations of ketorolac were extremely low after intravitreal and suprachoroidal administrations, suggesting that systemic adverse effects from the drug would be minimal. ${ }^{37}$

Safety data from the phenylephrine/ketorolac combination have been collected until postoperative day 90 in published clinical trials ${ }^{16,47,48}$ and have been constantly reported to be safe and well tolerated by patients. ${ }^{47}$

Treatment-emergent adverse effects reported in these trials included eye pain, blurred vision headache, ocular discomfort, and photophobia, and these were lower in patients treated with the combination than those in the placebo group. ${ }^{48}$ Other reported events that were equally prevalent in the treatment and placebo groups were posterior capsule opacification, corneal edema, eye inflammation, conjunctival hyperemia, and foreign body sensation. Only an increase in the intraocular pressure was reported to occur in a slightly greater proportion in patients in the treatment group $;{ }^{48}$ however, it was not statistically significant and the majority of the cases resolved within a few days and the event was not considered to be caused by treatment. ${ }^{26}$

\section{Patient-focused perspectives such as quality of life and patient satisfaction/acceptability}

As we discussed previously, cataract is the leading cause of treatable blindness worldwide and is associated with considerable disability and major economic and social consequences. ${ }^{5}$ Global population growth and increasing longevity are expected to double the number of people with visual loss from cataracts by the year 2020. ${ }^{56}$ Restoration of vision, through phacoemulsification cataract surgery, has been demonstrated to enhance quality of life as well as to improve household economic status. ${ }^{57}$ After surgery, people are more likely to undertake and spend more time on productive activities and less likely to report receiving assistance with daily life activities. ${ }^{57}$

Large studies have demonstrated that over the past decades, there has been an important change in practice to operating on eyes with less visual impairment. ${ }^{6}$ This might be explained by the improvement of surgical techniques and outcomes, patients' visual expectations, and a need for higher visual function to maintain independence. ${ }^{6}$ However, cataract surgery does not always result in an improvement in visual acuity or patient satisfaction, especially in patients with good preoperative visual acuity. ${ }^{6}$ A good preoperative self-assessed visual function has been demonstrated to be associated with poor patient-reported outcome measurements. ${ }^{58}$ This might be a problem of more developed countries where healthy people with minimum disability are offered the possibility of surgery ${ }^{58}$ together with the increasing demand of RLE., ${ }^{9,58}$ Clarifying the indications for cataract surgery and providing patients with thorough information before and after surgery can significantly improve the quality of care for these patients. ${ }^{58}$

Other important factors that negatively affect visual acuity and patients' reported surgical outcomes include the presence of ocular co-morbidities and complications during surgery. ${ }^{6,58,59}$ As we have mentioned earlier, posterior capsule rupture significantly increases the cost of surgery and is also associated with a higher risk of retinal detachment and endophthalmitis. ${ }^{6,20}$ Identifying risk factors preoperatively may minimize the risk of capsule complications. ${ }^{58}$ This and other serious complications are associated with poor pupil dilation $^{18}$ and measures should be taken to address this problem efficiently in order to prevent adverse events.

Although modern cataract surgery is considered to be a minor procedure with an uneventful and pain-free recovery period, moderate or severe postoperative pain has been reported to be relatively common after cataract surgery. ${ }^{41}$ 
Moreover, pain control intraoperatively increases patient cooperation, which is very important as topical anesthesia is currently preferred over more invasive methods. ${ }^{40}$

\section{Conclusion}

Cataracts are a global problem that cause important limitations to the patient's activities and quality of life. However, they can be treated with a safe and minimally invasive surgery. Improvements in surgical techniques and IOLs have greatly improved visual outcomes and thus patients' expectations regarding visual recovery are also increasing. As in every medical procedure, prevention is the best way to approach complications that can jeopardize refractive outcome or significantly increase the cost of the surgery. An adequate mydriasis is essential to safely perform cataract surgery.

There are many ways to achieve pupil dilation. Among the pharmacological tools, phenylephrine and ketorolac have demonstrated their efficacy in achieving and maintaining pupil dilation, as well as to control pain and inflammation in the postoperative period. The combination of phenylephrine and ketorolac has been shown to be a good pharmacological tool that combines the positive effects of both drugs to maintain intraoperative mydriasis, prevent surgery-induced miosis, and reduce postoperative pain and inflammation after cataract surgery. Furthermore, clinical trials report a good safety profile and patient tolerability. Moreover, recent evidence suggests that this combination might also be effective in patients with poor pupil dilation with the advantage that mydriasis can be achieved with no modifications to the surgical procedure.

\section{Disclosure}

The authors report no conflicts of interest in this work.

\section{References}

1. Gupta VB, Rajagopala M, Ravishankar B. Etiopathogenesis of cataract: an appraisal. Indian J Ophthalmol. 2014;62(2):103-110.

2. Truscott RJ, Friedrich MG. The etiology of human age-related cataract. Proteins don't last forever. Biochim Biophys Acta. 2016;1860(1 Pt B): 192-198.

3. Shah M, Shah S, Upadhyay P, Agrawal R. Controversies in traumatic cataract classification and management: a review. Can J Ophthalmol. 2013;48(4):251-258.

4. Lawrence D, Fedorowicz Z, van Zuuren EJ. Day care versus inpatient surgery for age-related cataract. Cochrane Database Syst Rev. 2015;11:CD004242.

5. Mohammadi SF, Hashemi H, Mazouri A, et al. Outcomes of cataract surgery at a referral center. J Ophthalmic Vis Res. 2015;10(3):250-256.

6. Day AC, Donachie PH, Sparrow JM, Johnston RL; Royal College of Ophthalmologists' National Ophthalmology Database. The Royal College of Ophthalmologists' National Ophthalmology Database study of cataract surgery: report 1, visual outcomes and complications. Eye (Lond). 2015;29(4):552-560.
7. Kim SJ, Schoenberger SD, Thorne JE, Ehlers JP, Yeh S, Bakri SJ. Topical nonsteroidal anti-inflammatory drugs and cataract surgery: a report by the American Academy of Ophthalmology. Ophthalmology. 2015;122(11):2159-2168.

8. Matta S, Park J, Palamaner Subash Shantha G, Khanna RC, Rao GN. Cataract surgery visual outcomes and associated risk factors in secondary level eye care centers of L V Prasad Eye Institute, India. PLoS One. 2016;11(1):e0144853.

9. Alio JL, Grzybowski A, El Aswad A, Romaniuk D. Refractive lens exchange. Surv Ophthalmol. 2014;59(6):579-598.

10. Shetty V, Haldipurkar SS, Gore R, Dhamankar R, Paik A, Setia MS. A comparison of visual outcomes in three different types of monofocal intraocular lenses. Int J Ophthalmol. 2015;8(6):1173-1178.

11. Vale C, Menezes C, Firmino-Machado J, et al. Astigmatism management in cataract surgery with Precizon $\left({ }^{\circledR}\right)$ toric intraocular lens: a prospective study. Clin Ophthalmol. 2016;10:151-159.

12. Lundström M, Manning S, Barry P, Stenevi U, Henry Y, Rosen P. The European registry of quality outcomes for cataract and refractive surgery (EUREQUO): a database study of trends in volumes, surgical techniques and outcomes of refractive surgery. Eye Vis (Lond). 2015;2(1):8.

13. Calladine D, Evans JR, Shah S, Leyland M. Multifocal versus monofocal intraocular lenses after cataract extraction. Sao Paulo Med J. 2015; 133(1):68

14. Rosen E, Alió JL, Dick HB, Dell S, Slade S. Efficacy and safety of multifocal intraocular lenses following cataract and refractive lens exchange: metaanalysis of peer-reviewed publications. J Cataract Refract Surg. 2016;42(2):310-328.

15. Alió JL, Vega-Estrada A, Plaza-Puche AB. Clinical outcomes with a new microincisional diffractive multifocal IOL. Eye Vis (Lond). 2015;2(1):2.

16. Grob S, Gonzalez-Gonzalez LA, Daly MK. Management of mydriasis and pain in cataract and intraocular lens surgery: review of current medications and future directions. Clin Ophthalmol. 2014;8:1281-1289.

17. Lee BS, Chang DF. Management of small pupils. Expert Rev Ophthalmol. 2016;11(1):49-58.

18. Hashemi H, Seyedian MA, Mohammadpour M. Small pupil and cataract surgery. Curr Opin Ophthalmol. 2015;26(1):3-9.

19. Lundström M, Behndig A, Montan A, et al. Capsule complication during cataract surgery: background, study design, and required additional care. J Cataract Refract Surg. 2009;35(10):1679-1687.e1.

20. Artzén D, Lundström M, Behndig A, Stenevi U, Lydahl E, Montan P. Capsule complication during cataract surgery: case-control study of preoperative and intraoperative risk factors: Swedish Capsule Rupture Study Group report 2. J Cataract Refract Surg. 2009;35(10):1688-1693.

21. Chu CJ, Johnston RL, Buscombe C, et al. Risk factors and incidence of macular edema after cataract surgery: a database study of 81984 eyes. Ophthalmology. 2016;123(2):316-323.

22. Muhtaseb M, Kalhoro A, Ionides A. A system for preoperative stratification of cataract patients according to risk of intraoperative complications: a prospective analysis of 1441 cases. Br J Ophthalmol. 2004; 88(10):1242-1246

23. Moreno-Montañés J, Sabater AL, Barrio-Barrio J, Pérez-Valdivieso JR, Cacho-Asenjo E, García-Granero M. Risks factors and regression model for risk calculation of anesthesiologic intervention in topical and intracameral cataract surgery. J Cataract Refract Surg. 2012;38(12):2144-2153.

24. Kardon R. Regulation of light through the pupil. Levin LA, Nilsson SFE, Ver Hoeve J, Wu SM, editors. In: Adler's Physiology of the Eye. New York: Elsevier; 2011:502-525.

25. Remington LA. Uvea. In: Clinical Anatomy and Physiology of the Visual System. St Louis: Elsevier/Butterworth-Heinemann; 2012:40-60.

26. Lawuyi LE, Gurbaxani A. The clinical utility of new combination phenylephrine/ketorolac injection in cataract surgery. Clin Ophthalmol. 2015;9:1249-1254.

27. Myers WG, Shugar JK. Optimizing the intracameral dilation regimen for cataract surgery: prospective randomized comparison of 2 solutions. $J$ Cataract Refract Surg. 2009;35(2):273-276. 
28. Saenz-de-Viteri M, Fernández-Robredo $\mathrm{P}$, de Nova E, et al. Comparative study measuring the dilatory effect of a mydriatic device (Mydriasert $\left({ }^{\circledR}\right)$ ) versus topical drops. Int J Ophthalmol. 2013;6(6): 801-804.

29. Lundberg B, Behndig A. Separate and additive mydriatic effects of lidocaine hydrochloride, phenylephrine, and cyclopentolate after intracameral injection. J Cataract Refract Surg. 2008;34(2): 280-283.

30. Lorente R, de Rojas V, Vazquez de Parga P, et al. Intracameral phenylephrine $1.5 \%$ for prophylaxis against intraoperative floppy iris syndrome: prospective, randomized fellow eye study. Ophthalmology. 2012;119(10):2053-2058.

31. Lundqvist $\mathrm{O}$, Koskela T, Behndig A. A paired comparison of intracameral mydriatics in refractive lens exchange surgery. Acta Ophthalmol. 2013;92(5):482-485.

32. Wilson DJ, Schutte SM, Abel SR. Comparing the efficacy of ophthalmic NSAIDs in common indications: a literature review to support costeffective prescribing. Ann Pharmacother. 2015;49(6):727-734.

33. Srinivasan R, Madhavaranga. Topical ketorolac tromethamine $0.5 \%$ versus diclofenac sodium $0.1 \%$ to inhibit miosis during cataract surgery. $J$ Cataract Refract Surg. 2002;28(3):517-520.

34. Chatterjee S. Preventing perioperative miosis in cataract surgery. $J$ Cataract Refract Surg. 2003;29(3):423.

35. Stewart R, Grosserode R, Cheetham JK, Rosenthal A. Efficacy and safety profile of ketorolac $0.5 \%$ ophthalmic solution in the prevention of surgically induced miosis during cataract surgery. Clin Ther. 1999; 21(4):723-732.

36. Holzer MP, Solomon KD, Sandoval HP, Vroman DT. Comparison of ketorolac tromethamine $0.5 \%$ and loteprednol etabonate $0.5 \%$ for inflammation after phacoemulsification: prospective randomized double-masked study. J Cataract Refract Surg. 2002;28(1):93-99.

37. Wang M, Liu W, Lu Q, et al. Pharmacokinetic comparison of ketorolac after intracameral, intravitreal, and suprachoroidal administration in rabbits. Retina. 2012;32(10):2158-2164.

38. Coelho RP, Biaggi RH, Jorge R, Rodrigues Mde L, Messias A. Clinical study of pain sensation during phacoemulsification with and without cryoanalgesia. J Cataract Refract Surg. 2015;41(4):719-723.

39. Eke T, Thompson JR. Serious complications of local anaesthesia for cataract surgery: a 1 year national survey in the United Kingdom. Br J Ophthalmol. 2007;91(4):470-475.

40. Jiang L, Zhang K, He W, Zhu X, Zhou P, Lu Y. Perceived pain during cataract surgery with topical anesthesia: a comparison between first-eye and second-eye surgery. J Ophthalmol. 2015;2015:383456.

41. Porela-Tiihonen S, Kaarniranta K, Kokki M, Purhonen S, Kokki H. A prospective study on postoperative pain after cataract surgery. Clin Ophthalmol. 2013;7:1429-1435.

42. Duong HV, Westfield KC, Chalkley TH. Ketorolac tromethamine LS $0.4 \%$ versus nepafenac $0.1 \%$ in patients having cataract surgery. Prospective randomized double-masked clinical trial. J Cataract Refract Surg. 2007;33(11):1925-1929.

43. Flach AJ, Dolan BJ, Irvine AR. Effectiveness of ketorolac tromethamine $0.5 \%$ ophthalmic solution for chronic aphakic and pseudophakic cystoid macular edema. Am J Ophthalmol. 1987;103(4):479-486.

44. Heier JS, Topping TM, Baumann W, Dirks MS, Chern S. Ketorolac versus prednisolone versus combination therapy in the treatment of acute pseudophakic cystoid macular edema. Ophthalmology. 2000; 107(11):2034-2038; discussion 2039.
45. Rho DS. Treatment of acute pseudophakic cystoid macular edema. J Cataract Refract Surg. 2003;29(12):2378-2384.

46. Weisz JM, Bressler NM, Bressler SB, Schachat AP. Ketorolac treatment of pseudophakic cystoid macular edema identified more than 24 months after cataract extraction. Ophthalmology. 1999;106(9):1656-1659.

47. Lindstrom RL, Loden JC, Walters TR, et al. Intracameral phenylephrine and ketorolac injection (OMS302) for maintenance of intraoperative pupil diameter and reduction of postoperative pain in intraocular lens replacement with phacoemulsification. Clin Ophthalmol. 2014;8:1735-1744.

48. Hovanesian JA, Sheppard JD, Trattler WB, et al. Intracameral phenylephrine and ketorolac during cataract surgery to maintain intraoperative mydriasis and reduce postoperative ocular pain: integrated results from 2 pivotal phase 3 studies. J Cataract Refract Surg. 2015; 41(10):2060-2068.

49. Rosenberg E, Alevi D, Donnenfeld E, Nattis A. Initial experience, visual outcomes, and efficiency of intracameral phenylephrine and ketorolac $(1.0 \% / 0.3)$ during cataract surgery. Paper presented at: the ASCRS Symposium on Cataract IOL and Refractive Surgery; May, 2016; New Orleans, LA

50. Visco D. Use of iris fixation ring with and without intracameral phenylephrine/ketorolac in patients with poor pupil dilation. Paper presented at: the ASCRS symposium on Cataract IOL and Refractive Surgery; May, 2016; New Orleans, LA.

51. Fry L. Effect of phenylephrine-ketorolac in maintaining mydriasis and intraoperative and first 24-hour comfort level during routine cataract surgery. Paper presented at: the ASCRS symposium on Cataract IOL and Refractive Surgery; May, 2016; New Orleans, LA.

52. Behndig A, Eriksson A. Evaluation of surgical performance with intracameral mydriatics in phacoemulsification surgery. Acta Ophthalmol Scand. 2004;82(2):144-147.

53. Bekir OA, Toufeeq S, Woods E, Jabir M. Effect of intracameral phenylephrine on systemic blood pressure. Eye (Lond). 2014;28(10): 1267-1268.

54. Mori Y, Miyai T, Kagaya F, et al. Intraoperative mydriasis by intracameral injection of mydriatic eye drops: in vivo efficacy and in vitro safety studies. Clin Experiment Ophthalmol. 2011;39(5):456-461.

55. Hong JW, Park JH, Kim ES, Kim JY, Kim MJ, Tchah H. Effect of intracameral injection of bisulfite-containing phenylephrine on rabbit corneal endothelium. Cornea. 2015;34(4):460-463.

56. Cabric E, Zvornicanin J, Jusufovic V. The safety and efficacy of day care cataract surgery. Med Arch. 2014;68(2):117-120.

57. Danquah L, Kuper H, Eusebio C, et al. The long term impact of cataract surgery on quality of life, activities and poverty: results from a six year longitudinal study in Bangladesh and the Philippines. PLoS One. 2014;9(4):e94140.

58. Mollazadegan K, Lundström M. A study of the correlation between patient-reported outcomes and clinical outcomes after cataract surgery in ophthalmic clinics. Acta Ophthalmol. 2014;93(3):293-298.

59. Macky TA, Mohamed AM, Emarah AM, Osman AA, Gado AS. Predicting postoperative visual outcomes in cataract patients with maculopathy. Indian J Ophthalmol. 2015;63(10):775-778.
Patient Preference and Adherence

\section{Publish your work in this journal}

Patient Preference and Adherence is an international, peer-reviewed, open access journal that focuses on the growing importance of patient preference and adherence throughout the therapeutic continuum. Patient satisfaction, acceptability, quality of life, compliance, persistence and their role in developing new therapeutic modalities and compounds to optimize

\section{Dovepress}

clinical outcomes for existing disease states are major areas of interest for the journal. This journal has been accepted for indexing on PubMed Central. The manuscript management system is completely online and includes a very quick and fair peer-review system, which is all easy to use. Visit http://www. dovepress.com/testimonials.php to read real quotes from published authors. 